CERN-ECP/96-08

30 July 1996

\title{
EXPERIMENTAL RESULTS ON RADIATION-INDUCED BULK DAMAGE EFFECTS IN FLOAT-ZONE AND EPITAXIAL SILICON DETECTORS
}

\author{
B. Dezillie, F. Lemeilleur, M. Glaser, G.-L. Casse $\left.{ }^{*}\right)$ \\ CERN, Geneva, Switzerland \\ C. Leroy \\ University of Montreal, Montreal, Canada
}

\begin{abstract}
A comparative study of the radiation hardness of silicon pad detectors, manufactured from float-zone and epitaxial n-type monocrystals and irradiated with protons and neutrons up to a fluence of $3.5 \times 10^{14} \mathrm{~cm}^{-2}$ is presented. The results are compared in terms of their reverse current, depletion voltage, and charge collection as a function of fluence during irradiation and as a function of time after irradiation.
\end{abstract}

Presented at the 5th International Workshop on Vertex Detectors, 16-21 June 1996, Chia, Cagliari, Italy.

*) Associazione per lo Sviluppo Scientifico e Tecnologico del Piemonte (ASP). 


\section{INTRODUCTION}

The silicon detectors to be used as active elements for the trackers placed close to the interaction points of the future high luminosity Large Hadron Collider (LHC) have to sustain over a period of 10 years a flux of the order of $10^{13} \mathrm{~cm}^{-2} \mathrm{yr}^{-1}$ of secondary particles produced by the head-on collisions of $7 \mathrm{TeV}$ protons. For this purpose, the radiation hardness of silicon detectors manufactured from high resistivity Float-Zone (FZ) refined silicon crystal material has been studied $[1,2]$. The results show that FZ silicon detectors can be operated up to hadron fluences of $10^{14} \mathrm{~cm}^{-2}$, at a temperature below $5^{\circ} \mathrm{C}$.

More recently, it was suggested [3] that the radiation hardness of silicon detectors depends on the impurity concentrations present in the initial silicon material. Parallel studies of silicon detectors produced from epitaxial (Epi) crystal-grown material were performed up to fluences of $\sim 10^{14}$ protons $\mathrm{cm}^{-2}$ [4]. As a result, safe operation of such detectors up to this fluence, at room temperature, was established. This work presents further results of FZ and epitaxial detectors irradiated up to proton and neutron fluences of $3.5 \times 10^{14} \mathrm{~cm}^{-2}$.

The experimental conditions, the irradiation facilities, and the measurement techniques are presented in Section 2. Results are given in Section 3.

\section{EXPERIMENTAL CONDITIONS}

\subsection{Test structures}

The characteristics of detectors are displayed in Table 1. It should be noted that one of the epitaxial detectors (C50) was processed from a different wafer than the other epitaxial detectors. Standard FZ diodes from n-type materials manufactured by Wacker and processed by Canberra and Micron Semiconductor typically have an area of $\sim 1 \mathrm{~cm}^{2}$ and a thickness of $\sim 300 \mu \mathrm{m}$.

\section{Table 1}

Characteristics of epitaxial detectors used in this study. $V_{\mathrm{fd}, 0}$ is the value of the full depletion voltage before irradiation.

\begin{tabular}{|c|c|c|c|c|c|c|}
\hline $\begin{array}{c}\text { Detector } \\
\text { name }\end{array}$ & Material & $\begin{array}{c}\text { Thickness } \\
(\mu \mathrm{m})\end{array}$ & $\begin{array}{c}\text { Area } \\
\left(\mathrm{cm}^{2}\right)\end{array}$ & $\begin{array}{c}\mathrm{V}_{\mathrm{fd}, 0} \\
(\mathrm{~V})\end{array}$ & $\begin{array}{c}\text { Resistivity } \\
(\mathrm{k} \Omega \mathrm{cm})\end{array}$ & $\begin{array}{c}\Phi \\
\mathrm{cm}^{-2}\end{array}$ \\
\hline \hline C50 & Epi & 120 & 0.82 & 48 & 0.94 & $1.3 \times 10^{14} \mathrm{p}$ \\
C62 & Epi & 110 & 0.82 & 43 & 0.86 & non-irr \\
C63 & Epi & 110 & 0.82 & 45 & 0.86 & $5.7 \times 10^{13} \mathrm{n}$ \\
C78 & Epi & 110 & 0.50 & 51 & 0.74 & $3.5 \times 10^{14} \mathrm{p}$ \\
C79 & Epi & 110 & 0.50 & 49 & 0.77 & $3.5 \times 10^{14} \mathrm{n}$ \\
M165 & FZ & 308 & 1 & 51 & 6 & non-irr \\
M160 & FZ & 306 & 1 & 49 & 6 & $3.5 \times 10^{14} \mathrm{p}$ \\
C28 & FZ & 307 & 0.82 & 189 & 1.57 & $1 \times 10^{14} \mathrm{p}$ \\
\hline
\end{tabular}

The n-type epitaxial diodes were manufactured by MACOM (USA) and processed by Canberra. The epitaxial layer is grown on a n-type Czochralski (CZ) base with a resistivity of $\sim 1 \Omega \mathrm{cm}$. 


\subsection{Irradiation facilities}

Proton irradiations were performed at the CERN Proton Synchroton (PS) using the PST7 $24 \mathrm{GeV} / c$ primary beam which produces several bursts per minute providing an average flux of $\sim 3 \times 10^{9} \mathrm{p} \mathrm{cm}^{-2} \mathrm{~s}^{-1}$.

The neutron irradiations were also performed at CERN using the Proton Synchroton Antiproton Irradiation Facility (PSAIF) which delivers bunches of $\sim 1 \mathrm{MeV}$ neutrons at a flux of $\sim 5 \times 10^{8} \mathrm{n} \mathrm{cm}^{-2} \mathrm{~s}^{-1}$.

\subsection{Measurement techniques}

Electrical characteristics were obtained from the current-voltage (IV) and capacitancevoltage (CV) measurements performed with a Keithley 237 High Voltage Source Measuring Unit and a Hewlett-Packard 4194A Impedance Analyzer operated at $10 \mathrm{kHz}$. The full depletion voltage is deduced from the measurement of capacitance versus voltage $(\mathrm{CV})$ curve and normalized to a $300 \mu \mathrm{m}$ diode thickness. The leakage current is obtained from the measurement of the current versus voltage (IV) curve at full depletion, normalized to $20^{\circ} \mathrm{C}$ and corrected for self-annealing.

After irradiation, the diodes were stored at $20^{\circ} \mathrm{C}$ and measured regularly during the first 100 days. After this time, they were heated for 1 to 3 hours at a maximum temperature of $90^{\circ} \mathrm{C}$, in order to accelerate the annealing process according to the model described in $[2,5]$.

Electrons from a ${ }^{106} \mathrm{Ru}$ source with energies greater than $2 \mathrm{MeV}$ (m.i.p.), selected by an external trigger, were used for charge collection measurements. The pulse induced by an electron in a diode is detected by a fast current amplifier (Phillips Scientific model 6954) and recorded on a digital oscilloscope operated in average mode.

\section{RESULTS}

\subsection{Leakage current}

\subsubsection{Leakage current versus fluence}

The change in volume leakage current with proton fluence is shown in Fig. 1. All data are normalized to $20^{\circ} \mathrm{C}$ and corrected for self-annealing, in order to compare measurements taken at different times after irradiation.

The leakage current damage constant, $\alpha$, is obtained from fitting the data to the form:

$$
\Delta I_{\mathrm{vol}}=\alpha \cdot \Phi
$$

where $\Delta I_{\mathrm{vol}}$ is the change in leakage current normalized to the depletion volume and $\Phi$ is the fluence. The fit to the epitaxial detector data yields an $\alpha$ value $\alpha_{\mathrm{C} 50}=(3.4 \pm 0.6) \times$ $10^{-17} \mathrm{~A} \mathrm{~cm}^{-1}$ for $\mathrm{C} 50$ irradiated with protons and $\alpha$ values of $\alpha_{\mathrm{C} 78}=(5.7 \pm 0.2) \times$ $10^{-17} \mathrm{~A} \mathrm{~cm}^{-1}$ and $\alpha_{\mathrm{C} 79}=(5.5 \pm 1.3) \times 10^{-17} \mathrm{~A} \mathrm{~cm}^{-1}$ for $\mathrm{C} 78$ and $\mathrm{C} 79$ irradiated with protons and neutrons, respectively. The discrepancy between the values obtained for C78, C79 and C50 could be due to the fact that this last detector was processed from a different epitaxial wafer. The values obtained for C78 and C79 are similar to the average value obtained for standard FZ detectors irradiated up to $1 \times 10^{14}$ particles $\mathrm{cm}^{-2}$ [6], where the $\alpha$ value is $\alpha_{\mathrm{FZ}}=(5.4 \pm 0.5) \times$ $10^{-17} \mathrm{~A} \mathrm{~cm}^{-1}$. 


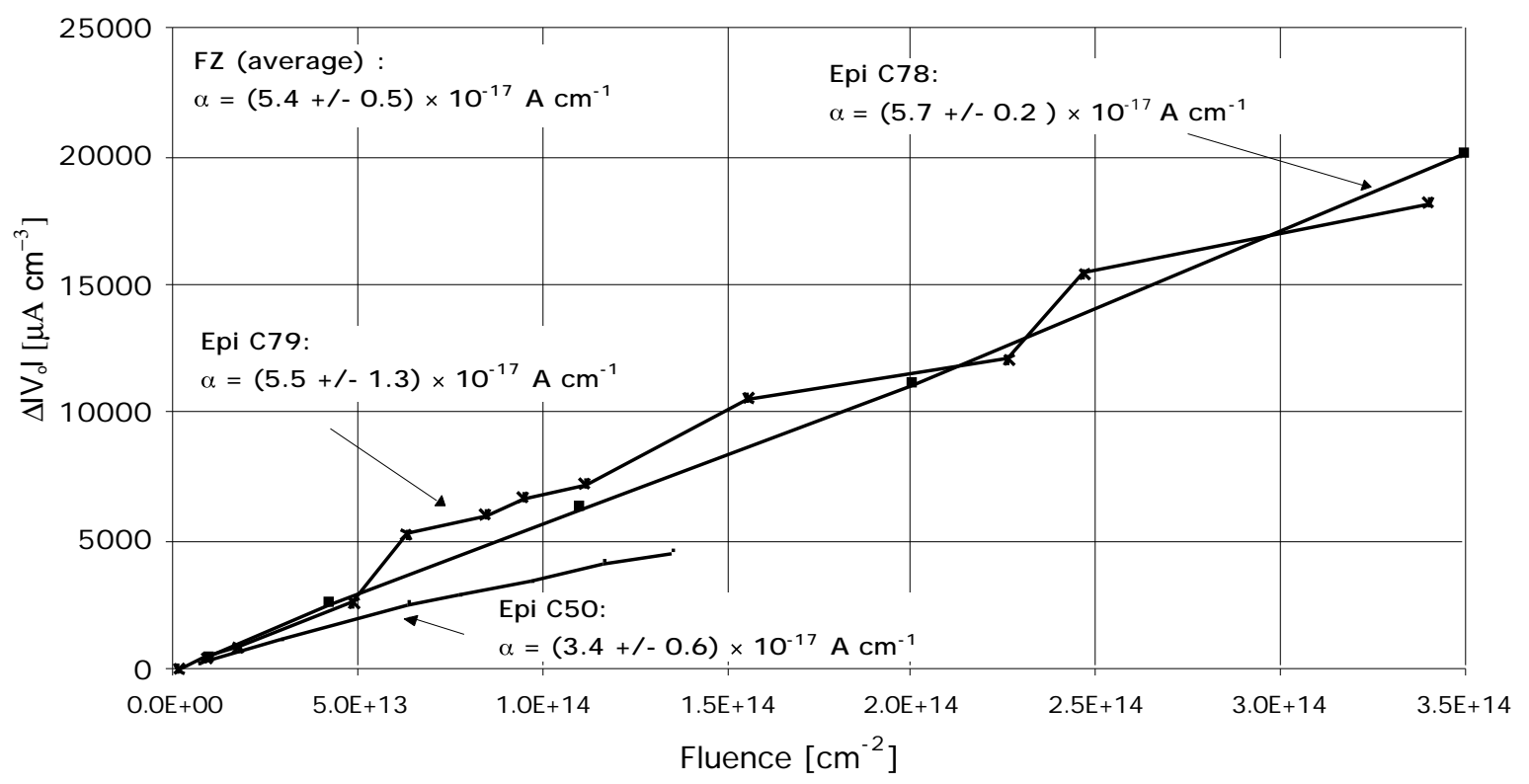

Fig. 1: The change in volume leakage current versus proton fluence for FZ and epitaxial detectors. The leakage current has been normalized to $20^{\circ} \mathrm{C}$ and corrected for self-annealing.

\subsubsection{Leakage current versus time after irradiation}

The behaviour of the leakage current with time after irradiation is shown in Fig. 2 for different epitaxial and standard FZ detectors. The value of $\alpha$ is shown rather than the leakage current to normalize for differences in various proton and neutron fluence values between the different detectors. Similarly to standard FZ diodes, the leakage currents of the epitaxial detectors decrease regularly to achieve $\alpha$ values close to $1 \times 10^{-17} \mathrm{~A} \mathrm{~cm}^{-1}$ after an equivalent time at room temperature corresponding to 10 years of $\mathrm{LHC}$ active time.

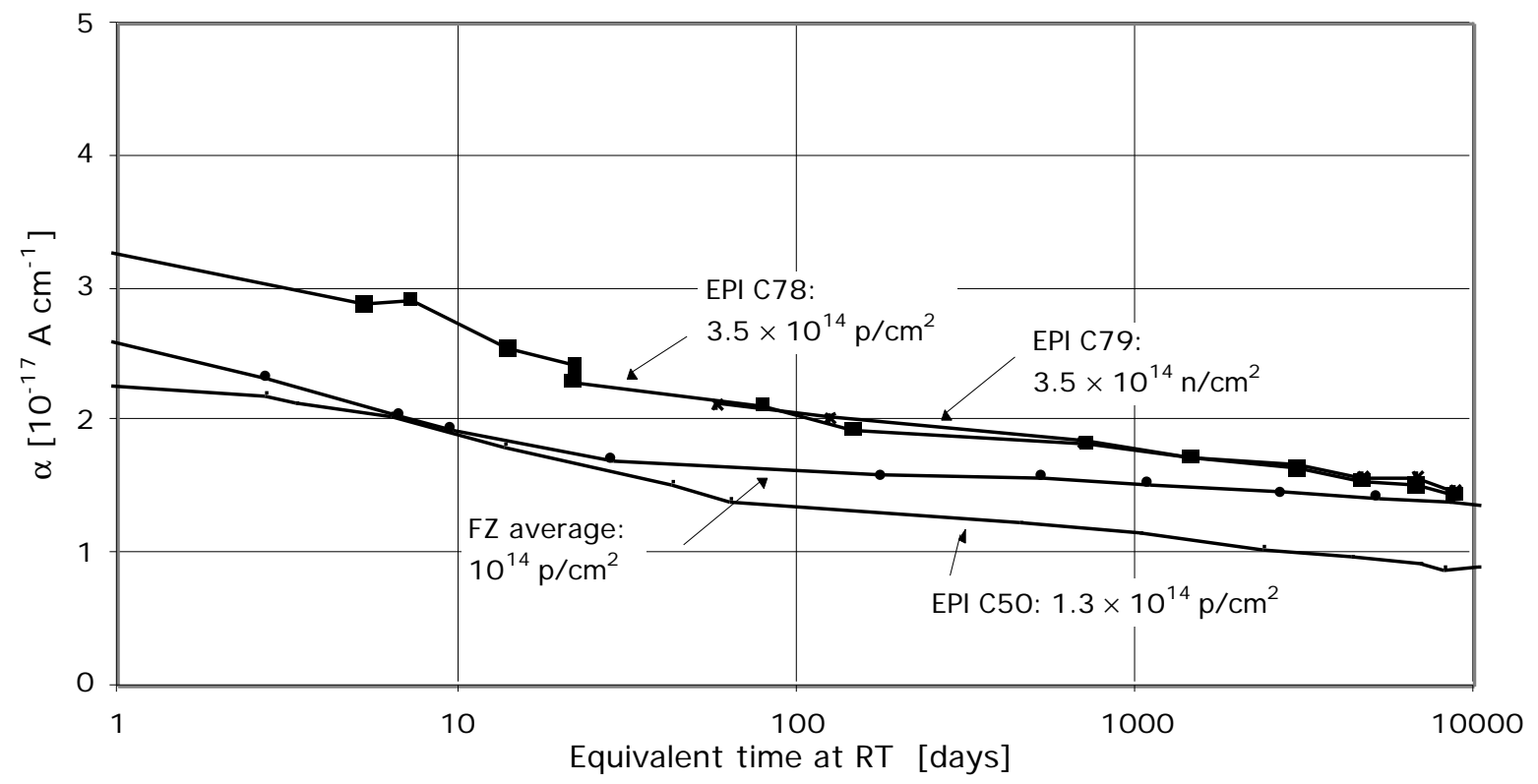

Fig. 2: Leakage current damage constant as a function of equivalent time at RT for FZ and epitaxial detectors. 


\subsection{Depletion voltage}

\subsubsection{Depletion voltage versus fluence}

Figure 3a shows the full depletion voltage normalized to a diode thickness of $300 \mu \mathrm{m}$ as a function of fluence for different epitaxial and standard FZ detectors. Measurements are taken within 1 hour after each irradiation step so that long-term annealing can be neglected.
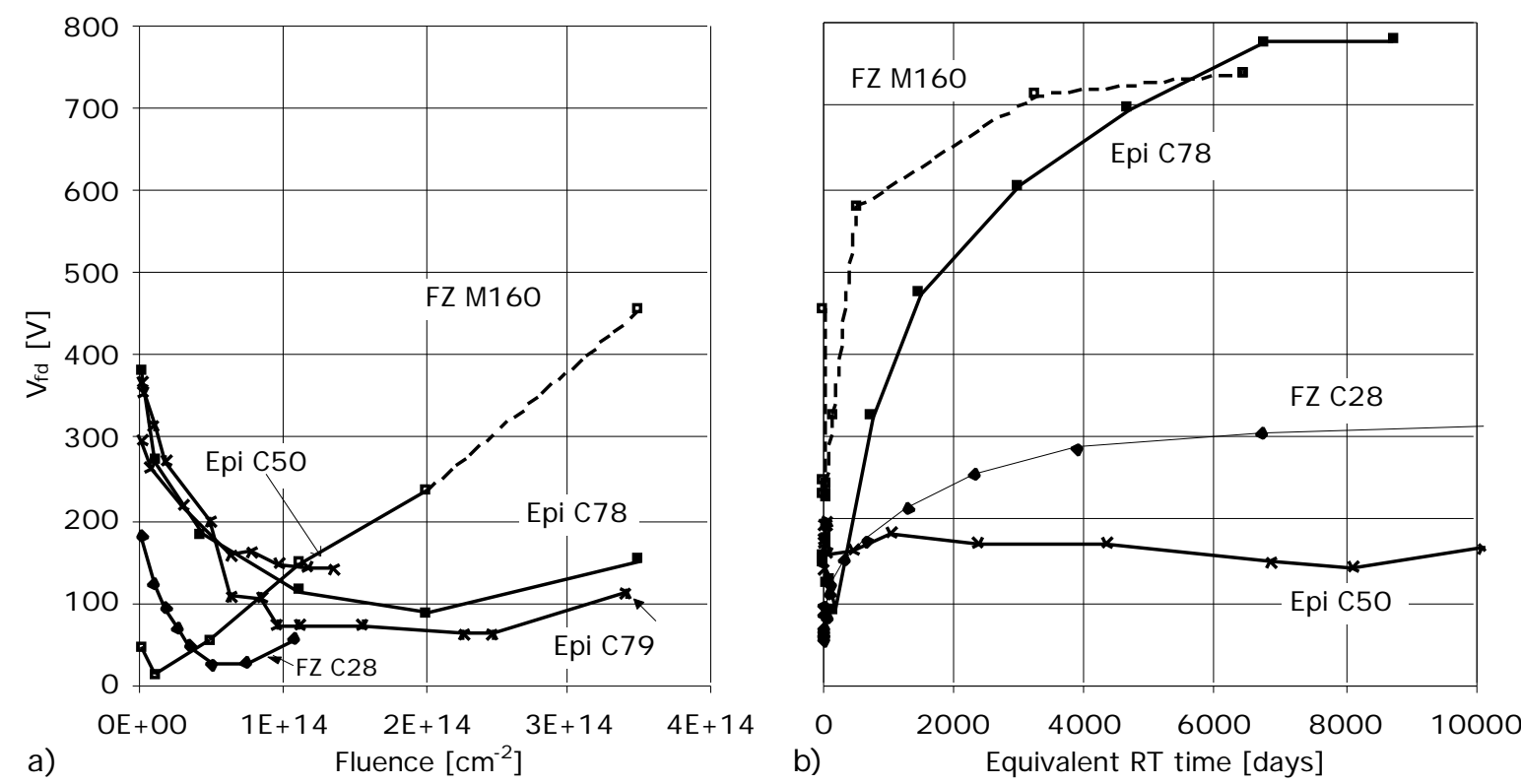

Fig. 3: Depletion voltage (normalized to a thickness of $300 \mu \mathrm{m}$ ) for $\mathrm{FZ}$ and epitaxial detectors as a function of (a) proton fluence and (b) equivalent time at room temperature after irradiation. No inversion is observed for epitaxial detectors before a proton fluence of $\sim 2 \times 10^{14} \mathrm{~cm}^{-2}$.

At a fluence of $1.3 \times 10^{14}$ protons $\mathrm{cm}^{-2}$, one epitaxial detector (C50) is not yet inverted [4], while the other epitaxial detectors irradiated up to $3.5 \times 10^{14}$ protons $\mathrm{cm}^{-2}(\mathrm{C} 78)$ and neutrons $\mathrm{cm}^{-2}$ (C79) show an inversion after a proton fluence of $\sim 2 \times 10^{14} \mathrm{~cm}^{-2}$. Using $\Phi_{\text {inv }}=$ (18 \pm 0.6$) N_{\text {eff, } 0}$ as established experimentally for FZ detectors [6], the inversion should have happened at $\sim 8 \times 10^{13} \mathrm{~cm}^{-2}$ for C50 and at $\sim 1 \times 10^{14} \mathrm{~cm}^{-2}$ for C78 and C79. The measured inversion point for the heavily irradiated epitaxial detectors are more than a factor 2 higher than the calculated value using the above equation.

\subsubsection{Effective doping concentration versus detector thickness}

Figure 4 shows the effective doping concentration $\left(N_{\text {eff }}\right)$ for an epitaxial detector $(\mathrm{C} 78)$ and a FZ detector (M160) as a function of their depletion depth deduced from the capacitance at the corresponding voltage. The doping concentration $\left(N_{\text {eff }}\right)$ is constant with the depletion thickness for the non-irradiated FZ detector (M160), while $N_{\text {eff }}$ is increasing for the non-irradiated epitaxial detector (C78). The resistivity $(\rho)$ which is determined as:

$$
\rho=1 / e \mu N_{\text {eff }}
$$

with $e$ the electron charge and $\mu$ the electron mobility, decreases when entering deeper into the bulk for the epitaxial detector. Measurements for the epitaxial detector show that the effective doping concentration before and after irradiation with $1.6 \times 10^{14}$ protons $\mathrm{cm}^{-2}$ has not changed in the CZ substrate, while it has decreased in the epitaxial layer $(\sim 110 \mu \mathrm{m})$. This method allows us to deduce the thickness of the epitaxial layer. 


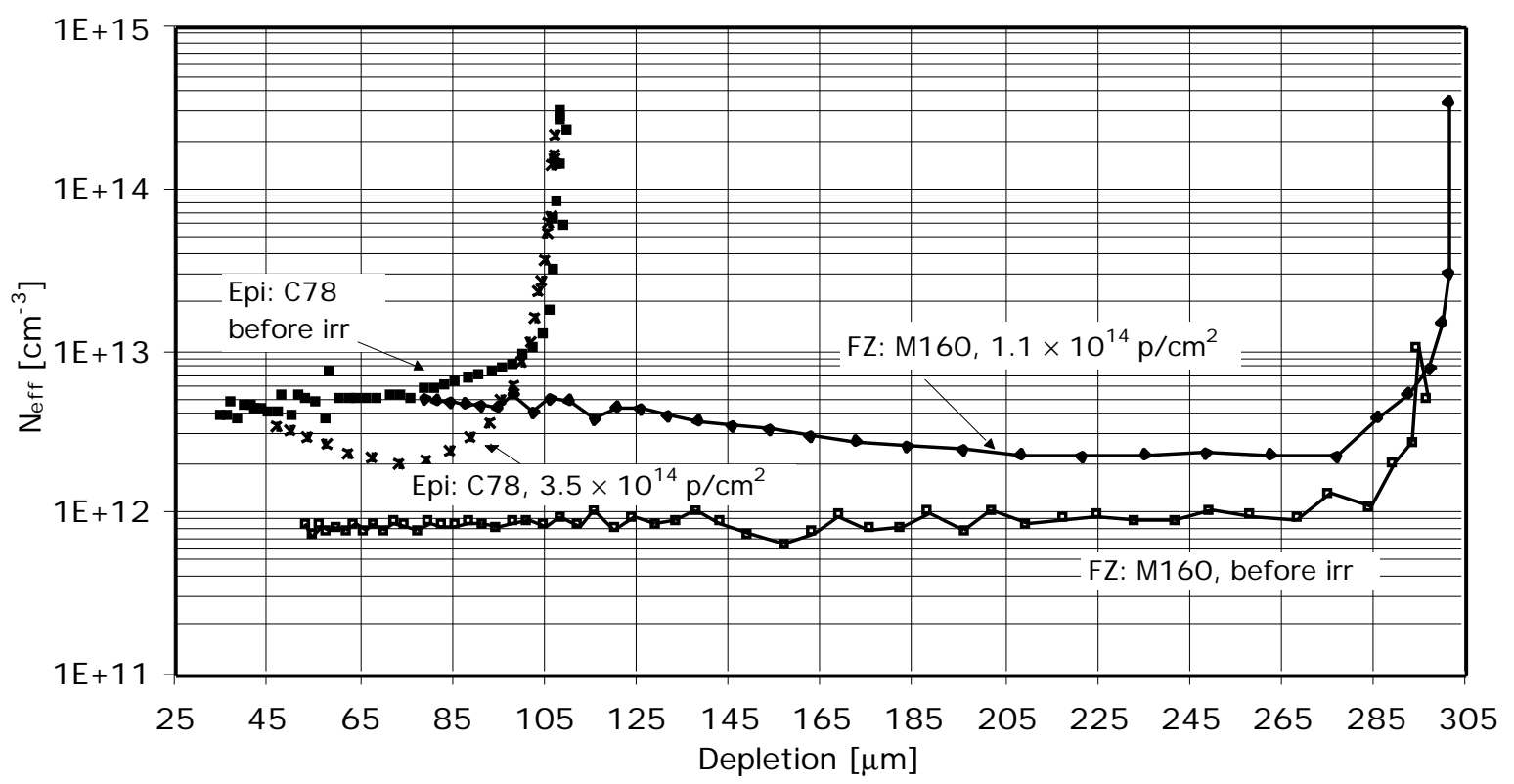

Fig. 4: The effective doping concentration $\left(N_{\text {eff }}\right)$ for a FZ and an epitaxial detector before and after irradiation as a function of detector thickness.

\subsubsection{Depletion voltage versus time after irradiation}

Figure $3 \mathrm{~b}$ shows the full depletion voltage (normalized to a thickness of $300 \mu \mathrm{m}$ ) as a function of equivalent time at room temperature for $\mathrm{FZ}$ and epitaxial detectors irradiated at $\sim 10^{14}$ and $3.5 \times 10^{14}$ protons $\mathrm{cm}^{-2}$ (see Table 1$)$. The non-inverted $\left(1.3 \times 10^{14}\right.$ protons $\mathrm{cm}^{-2}$, see Fig. 3a) epitaxial detector (C50) shows no reverse annealing. The process of reverse annealing for non-inverted $\mathrm{FZ}$ detectors has already been studied as a function of equivalent time at RT [4]. A strong reverse annealing is observed for the epitaxial detector (C78), irradiated to the level of $3.5 \times 10^{14}$ protons $\mathrm{cm}^{-2}$ and tends to saturate at a full depletion voltage of $\sim 800 \mathrm{~V}$ after 9000 days equivalent time at RT. For comparison, a FZ detector (M160) irradiated at the same fluence level, is presented (dashed line) on the same figure. For this detector, however, the full depletion voltages could not be measured anymore after a fluence of $\sim 2 \times 10^{14}$ protons $\mathrm{cm}^{-2}$, because of a 'current runaway'. The full depletion voltages were deduced from extrapolation of the capacitance values measured at lower voltages.

These results of non-inversion of an epitaxial detector before a fluence of $\sim 2 \times 10^{14}$ particles $\mathrm{cm}^{-2}$ and a constant value of the full depletion voltage during the annealing process for a non-inverted epitaxial diode are important for the future LHC experiments. Figure 5 shows, as a function of the $\mathrm{LHC}$ operation time, $1 \mathrm{MeV}$ equivalent neutron fluence at representative radii, $10 \mathrm{~cm}, 20 \mathrm{~cm}, 30 \mathrm{~cm}$ and $50 \mathrm{~cm}$. This roughly corresponds to the ATLAS/CMS inner pixel layer, the CMS inner strip layer, the ATLAS inner strip layer, and the ATLAS/CMS outer strip layer, respectively [7]. After 10 years of operation, epitaxial detectors could still be used fully depleted at voltages $\leq 50 \mathrm{~V}$ for a thickness of $110 \mu \mathrm{m}$ in the whole radius range of the LHC trackers, with the exception of the ATLAS/CMS inner pixel layer. 
Fig. 5: $1 \mathrm{MeV}$ equivalent neutron fluence at 50,30,20 and $10 \mathrm{~cm}$ radii, roughly corresponding to the ATLAS/CMS inner pixel layer, the CMS inner strip layer, the ATLAS inner strip layer, and the ATLAS/CMS outer strip layer, respectively, for worst-case assumptions [7].

\subsection{Charge collection}

Figure 6 shows as a function of $V^{1 / 2}$ the average charge collection from signal induced by relativistic electrons in non-irradiated and irradiated $\left(3.5 \times 10^{14}\right.$ protons $\left.\mathrm{cm}^{-2}\right) \mathrm{FZ}(\mathrm{M} 165$ and M160, respectively) and epitaxial (C62 and C78, respectively) silicon detectors.

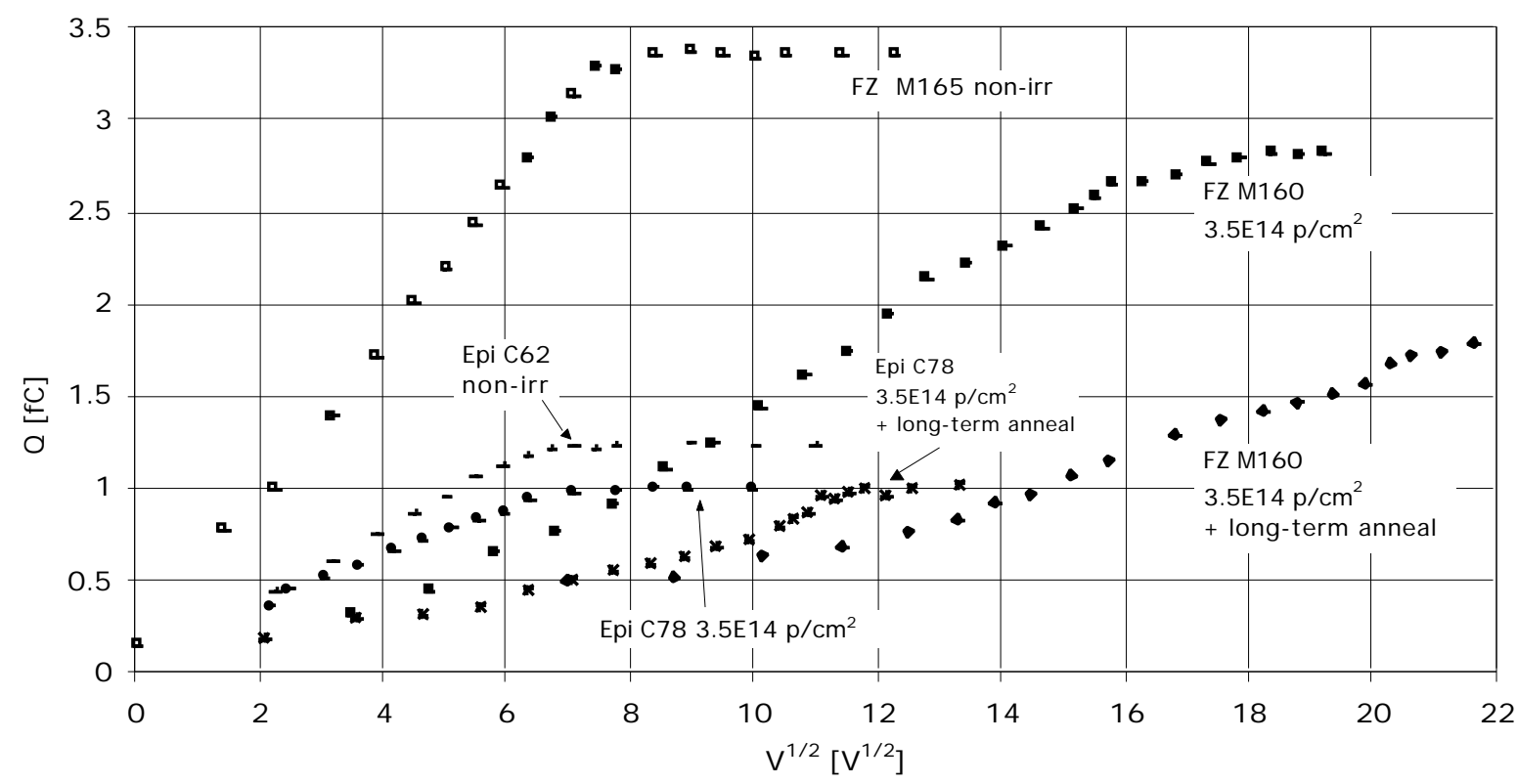

Fig. 6: The charge collection as a function of $V^{1 / 2}$ for non-irradiated, irradiated, and long-term annealed FZ and epitaxial detectors.

For non-irradiated detectors, the saturations are achieved at and above the full depletion voltages as measured from the capacitance-versus-voltage (CV) curves. The saturation values are in the ratio of the detector thicknesses, $308 \mu \mathrm{m}$ for FZ and $110 \mu \mathrm{m}$ for epitaxial. For the epi- 
taxial detector (C78), the charge collection efficiency after $3.5 \times 10^{14}$ protons $\mathrm{cm}^{-2}$ and beyond the full depletion voltage is $81.2 \pm 3.2 \%$. The same efficiency is obtained after long-term annealing, although, a higher voltage is required by the reverse annealing process.

For the FZ detector (M160), a similar efficiency of $82 \pm 4 \%$ is achieved at the same fluence just after irradiation. However, after long-term annealing, the charge collection could not be measured beyond $450 \mathrm{~V}$ due to reverse current runaway. This voltage is too low to fully deplete the detector. A linear extrapolation of the data gives a full depletion voltage value of $\sim 900 \mathrm{~V}$.

\section{CONCLUSION}

Epitaxial detectors (n-type) with a resistivity of 700-900 $\Omega \mathrm{cm}$ and a thickness of 110 $120 \mu \mathrm{m}$ have been studied and compared with standard (n-type) FZ detectors. They have been irradiated at room temperature with $1 \mathrm{MeV}$ neutrons and $24 \mathrm{GeV} / c$ protons. The value of the reverse current damage constant corrected for self-annealing during the proton and neutron irradiation for two epitaxial detectors processed from the same wafer was found to be $\alpha=(5.6 \pm$ 1.3) $\times 10^{-17} \mathrm{~A} \mathrm{~cm}^{-1}$, which is similar to the average value obtained for standard FZ detectors.

A smaller value $\left[\alpha_{\mathrm{C} 50}=(3.4 \pm 0.6) \times 10^{-17} \mathrm{~A} \mathrm{~cm}^{-1}\right]$ was obtained for another epitaxial detector processed from another wafer.

After long-term annealing at equivalent room temperature, the reverse current decreases also similarly to standard FZ detectors, achieving an $\alpha$ value of $\sim 10^{-17} \mathrm{~A} \mathrm{~cm}^{-1}$.

The measured type inversion for the epitaxial detectors occurs above a fluence of $\sim 2 \times$ $10^{14}$ particles $\mathrm{cm}^{-2}$, which is more than a factor 2 higher than the predicted value for the FZ material with the same resistivity. Higher impurity concentrations such as carbon and oxygen introduced during the epitaxial silicon crystal growing process could be the reason for the improved radiation hardness of epitaxial detectors compared to FZ detectors.

This study proves that there exists silicon material such as epitaxial from which detectors can be manufactured. Such detectors can be operated fully depleted at room temperature at voltages $<50 \mathrm{~V}$ for a thickness of $110 \mu \mathrm{m}$ up to a fluence of $\sim 2 \times 10^{14}$ particles $\mathrm{cm}^{-2}$.

The charge collection efficiency for epitaxial detectors with a deficit of $\sim 18 \%$ after a proton fluence of $3.5 \times 10^{14} \mathrm{~cm}^{-2}$ is similar to the deficit obtained for FZ detectors.

This surprising behaviour during and after irradiation of diodes processed from epitaxial grown crystals is a confirmation that the radiation hardness of silicon depends on the impurity concentrations of the initial material [3]. Measurements of the impurity concentrations of the FZ and epitaxial material used for this study are still under way. New measurements are foreseen with epitaxial detectors with a higher resistivity and a larger thickness. A production of FZ silicon crystals with different impurity concentrations is also under way.

\section{Acknowledgements}

The authors would like to thank E. Heijne from the RD19 Collaboration for initiating the use of epitaxial material, Canberra for providing epitaxial samples, and S. Watts for helpful discussions. The work of L. Durieu in preparing the proton beam for the irradiations is also greatly appreciated. 


\section{References}

[1] F. Lemeilleur et al., 'Electrical properties and charge collection efficiency for neutronirradiated p-type and n-type silicon detectors', Nucl. Phys. B (Proc. Suppl.) 32 (1993) 415.

[2] E. Fretwurst et al., 'Radiation hardness of silicon detectors for future colliders', Nucl. Intrum. Methods A326 (1993) 357.

[3] J. Matheson et al., 'The effect of radiation defects on the performance of high resistivity silicon diodes', RD20/TN/36 (1995).

[4] B. Dezillie et al., 'Radiation hardness of silicon detectors manufactured on wafers from various sources', CERN-ECP/96-06, to appear in the Proceedings of the International Conference on Radiation Effects on Semiconductor Materials, Detectors and Devices, 6-8 March 1996, Florence, Italy.

[5] A. Chilingarov et al., Radiation studies and operational projections for silicon in the ATLAS inner detector, Nucl. Instrum. Methods A360 (1995) 432.

[6] S.J. Bates et al., 'Proton irradiation of silicon detectors with different resistivities', CERN-ECP/95-18 and Proceedings of the IV International Workshop on Vertex Detectors, 11-16 June 1995, Ein Gedi Resort, Dead Sea, Israel.

[7] CMS TN/95-198. 